\title{
F1 Offspring of (F0) Female Rats Fed a High-Saturated Fat, Prenatal/Lactation Diet Remain Insulin Resistant Despite Postnatal Diet Rich in 0mega-3 Polyunsaturated Fatty Acids
}

\author{
Julie J. Kachinski', Hongbin Jin², Daniel C. Benyshek ${ }^{1}$ \\ ${ }^{1}$ Metabolism, Anthropometry and Nutrition Laboratory, University of Nevada, Las Vegas, Las Vegas, USA \\ ${ }^{2}$ Department of Epidemiology and Biostatistics, University of Nevada, Las Vegas, Las Vegas, USA \\ Email: daniel.benyshek@unlv.edu
}

Received 15 November 2014; revised 5 December 2014; accepted 15 December 2014

Copyright (C) 2014 by authors and Scientific Research Publishing Inc.

This work is licensed under the Creative Commons Attribution International License (CC BY).

http://creativecommons.org/licenses/by/4.0/

(c) (i) Open Access

\begin{abstract}
Prior research has shown adult diets rich in omega-3 long-chain polyunsaturated fatty acids (omega-3 LC-PUFAs) can improve adult metabolic health. Previous studies have also shown maternal overnutrition during pregnancy/lactation adversely affects metabolic functioning in adult offspring. The purpose of the current study was to investigate the interaction of these two metabolism regulating factors by assessing the effectiveness of a postweaning diet rich in omega-3 long chain-polyunsaturated fatty acids (omega-3 LC-PUFAs) to improve metabolic function in adult offspring whose mothers were fed a high-saturated fat "Western" diet during pregnancy/lactation. We compared metabolic function between offspring of three prenatal-lactation/postweaning diet lines of Sprague-Dawley rats: 1) offspring of mothers fed a high-saturated fat "Western" diet during pregnancy-lactation, then weaned to a high omega-3 LC-PUFA diet (Western/PUFA); 2) off- spring of mothers fed a control diet during pregnancy-lactation, then weaned to a high omega-3 LC-PUFA diet (Control/PUFA); and 3) offspring of mothers fed a Western diet during pregnancy- lactation, and postweaning (Western/Western). Fasting plasma insulin, triglycerides, and insulin resistance (HOMA-IR) of Western/PUFA animals were intermediate to those of Western/Western and Control/PUFA offspring, although these differences did not reach statistical significance. This suggests the metabolic benefits of an omega-3 LC-PUFA-rich diet are insufficient to overcome the deleterious effects of a high-saturated fat prenatal-lactation diet.
\end{abstract}

\section{Keywords}

Developmental Origins, Omega-3 LC-PUFAs, Insulin Resistance, Animal Modelling 


\section{Introduction}

The health benefits associated with a diet rich in omega-3 fatty acids, and/or a low omega-6:omega-3 ratio, have been reported in a large number of epidemiological and clinical studies. The cardiometabolic benefits associated with these diets include reduced insulin resistance, improved blood lipid profiles, lowered blood pressure and decreased incidence of type 2 diabetes and metabolic syndrome [1]-[3]. Experimental animal studies have complemented for these epidemiological and clinical findings in humans, reporting similar improvements regarding inflammation, plasma triglycerides, cholesterol, insulin sensitivity, and adipocyte insulin stimulated by glucose transport in adult animals fed diets rich in omega 3 fatty acids and/or with a low omega-6:omega-3 ratio [4]-[7].

Epidemiological and experimental animal research has also shown maternal overnutrition during pregnancy and lactation, especially via a high energy, high-saturated fat, low fiber "Western" diet, deleteriously alters metabolic function in adult offspring, even when animals are weaned onto control diets [8]. Given the widely acknowledged role of high energy, high fat, low fiber "Western" diets in the global obesity and type 2 diabetes pandemic [9], the purpose of the current study was to investigate the effect of a high-saturated fat Western prenatal-lactation diet on the effectiveness of a postweaning diet rich in omega-3 LC-PUFAs to improve the metabolic profile of mature offspring.

\section{Materials and Methods}

Sprague-Dawley male and female adult breeding animals were acquired from Simonsen Laboratories, Inc., and housed at the University of Nevada Las Vegas Animal Care Facility. During a 10-day acclimatization period, male and female animals were kept in separate plastic cages and fed a standard chow diet (Table 1). Female animals were then randomly assigned to either a "Western" or "Control" diet on day 10. The Western diet was formulated to model a high fat, highly processed "fast food” diet, typical of the global nutritional transition [9]. A standard rat chow was used for the Control diet (Table 2). Male breeders were housed separately, and, like the female breeders, food and water were supplied ad libitum. After seven days on their assigned diets, female animals were placed in larger cages with males to breed. Male breeders were introduced into larger cages with dams fed the Control diet after the females consuming the Western diet became pregnant. Male animals consumed the same diet as their cage mates. Litters from each prenatal diet group were standardized for sex and litter size using culling and cross-fostering techniques when the pups were 10 days old. At weaning, pups were fed one of two diets: a balanced omega-6/omega-3 LC-PUFA-rich diet (PUFA) or the high-saturated fat diet (Western) (Table 2). Animals were weighed approximately every third day for 120 days. On the 120th day, animals were fasted overnight. That morning, after being restrained in a breathable tube, lidocaine was applied to

Table 1. Experimental Design.

\begin{tabular}{ccc}
\hline Fo Generation & F1 Generation \\
\hline Maternal Diets & Post-weaning Diets \\
\hline Control & PUFA \\
Western & PUFA \\
Western & Western \\
\hline
\end{tabular}

Table 2. Diet Composition.

\begin{tabular}{lccc}
\hline Component & Control & PUFA & Western \\
\hline Calories provided by & & & \\
Protein & $28.5 \%$ & $30.3 \%$ & $17.8 \%$ \\
Fat & $13.5 \%$ & $59.8 \%$ & $29.8 \%$ \\
Carbohydrate & $58.0 \%$ & $10.0 \%$ & $52.3 \%$ \\
P:S & $1: 1$ & $2: 1$ & $0.5: 1$ \\
Omega-6:Omega-3 & $6.5: 1$ & $1.5: 1$ & $9: 1$ \\
\hline
\end{tabular}


approximately $1 \mathrm{~cm}$ of the end of the tail. Approximately $2 \mathrm{~mm}$ of the tip of the tail was excised with a surgical scalpel, and blood was collected by milking the tail. Heparin coated capillary tubes were used to collect approximately $100 \mu \mathrm{l}$ of blood for glucose and blood lipid analyses. In addition, $600 \mu \mathrm{l}$ of blood was collected in non-heparin capillary tubes for insulin analysis. These samples were spun at $4000 \mathrm{~g}$ at $4^{\circ} \mathrm{C}$, after which the plasma was removed from the samples and stored in cryogenic vials at $-40^{\circ} \mathrm{C}$ until insulin analysis was performed. The study was approved by the UNLV Institutional Animal Care and Use Committee (IACUC).

\subsection{Experimental Diets}

Study diets varied in terms of macronutrients, sources of fat and polyunsaturated to saturated fatty acid ratios (Table 2). Although macronutrient content of the PUFA diet (Purina Test diet, Greenfield, Indiana) was identical for breeders and weanlings (30\% protein, 10\% carbohydrate, and 60\% fat), the mineral content of the diet for breeders contained slightly more iron, zinc, selenium and calcium, as recommended by the commercial vendor nutritionist. Fat sources for the PUFA diet were soybean oil and fish (Table 2). Western diet fat sources were a mixture of cocoa butter, milk-fat, beef tallow, lard, and fish, coconut, safflower, soybean, canola and corn oils (Harlan Teklad Madison, Wisconsin). PUFA and Western diets both contained a standardized AIN-93 vitamin and mineral mixture. Control diets were stored at room temperature, Western diets were kept at $4^{\circ} \mathrm{C}$, and PUFA diets were stored at $-40^{\circ} \mathrm{C}$. Each week, one week's supply of PUFA chow would be transferred and kept at $4^{\circ} \mathrm{C}$ until being dispensed to cages. Animals consuming the PUFA diet were supplied with fresh food every two days to prevent excess oxidation of the chow. Western diets were supplied with fresh food every three days. Control diets were continuously resupplied.

\subsection{Biomarker Analyses}

Analysis of plasma blood glucose and lipids were performed using the Abaxis Blood Chemistry Analyzer. Glycosylated hemoglobin (HbA1c) was measured using the Bayer DCA 200 Chemistry Analyzer. Insulin was analyzed via ELISA by ZRT Laboratories in Beaverton, Oregon.

\subsection{Statistical Methods}

The three groups are not independent of each other given that the Western/PUFA animals shared maternal diet with the Western/Western animals and postweaning diet with the Control/PUFA animals. As a result, MannWhitney U tests were used to examine differences in body weight/BMI, HbA1c, fasting plasma glucose, HOMAIR, fasting plasma insulin, and triglycerides among the three experimental diet groups. For body weight data, given a larger sample size, 2 sample t-tests were used to examine differences in body weight among pre-weaning pups after data normality was confirmed. Levene's tests were performed to examine the homogeneity of variances. Data were analyzed using SPSS version 21.0 (IBM Corp., Armonk, NY). Descriptive statistics were used to detect potential outliers of which values were greater or less than mean \pm 1.65 standard deviations. The significance level was fixed at 0.05 for all statistical tests.

\section{Results}

Table 3 shows the details of the metabolic markers for offspring of the three diet lines: Control/PUFA, Western/ PUFA and Western/Western. Table 4 provides data on statistical comparisons of biomarkers for the offspring of the three diet lines.

\subsection{Glucose}

Mean fasting glucose was lower among Western/Western animals than both Control/PUFA and Western/PUFA offspring, although these differences were not statistically significant. Mean HbA1c values varied minimally among Control/PUFA, Western/PUFA and Western/Western animals and did not reach statistical significance.

\subsection{Insulin}

Mean fasting insulin and HOMA-IR values of Western/PUFA animals were intermediate to those of Western/ Western and Control/PUFA offspring. While mean plasma insulin was 23\% lower, and mean HOMA-IR was $10 \%$ 
Table 3. Metabolic markers for male and female post-weaning rats by experimental diet.

\begin{tabular}{|c|c|c|c|c|c|c|}
\hline \multirow{2}{*}{ Biomarker } & \multicolumn{2}{|c|}{ Control/PUFA } & \multicolumn{2}{|c|}{ Western/PUFA } & \multicolumn{2}{|c|}{ Western/Western } \\
\hline & $\mathrm{N}$ & Mean \pm S.E. & $\mathrm{N}$ & Mean \pm S.E. & $\mathrm{N}$ & Mean \pm S.E. \\
\hline $\operatorname{BMI}\left(\mathrm{g} / \mathrm{cm}^{2}\right)$ & 5 & $0.60 \pm 0.02$ & 4 & $0.61 \pm 0.04$ & 3 & $0.60 \pm 0.04$ \\
\hline Male & 3 & $0.62 \pm 0.02$ & 2 & $0.68 \pm 0.01$ & 2 & $0.65 \pm 0.01$ \\
\hline Female & 2 & $0.57 \pm 0.02$ & 2 & $0.55 \pm 0.02$ & 1 & $0.52 \pm 0.00$ \\
\hline HbA1c (\%) & 5 & $3.38 \pm 0.09$ & 4 & $3.35 \pm 0.03$ & 3 & $3.37 \pm 0.03$ \\
\hline Male & 3 & $3.50 \pm 0.06$ & 2 & $3.35 \pm 0.05$ & 2 & $3.40 \pm 0.00$ \\
\hline Female & 2 & $3.20 \pm 0.10$ & 2 & $3.35 \pm 0.05$ & 1 & $3.30 \pm 0.00$ \\
\hline Glucose (mmol/L) & 5 & $8.57 \pm 0.45$ & 4 & $8.61 \pm 0.34$ & 3 & $7.48 \pm 0.27$ \\
\hline Male & 3 & $8.63 \pm 0.75$ & 2 & $8.92 \pm 0.08$ & 2 & $7.75 \pm 0.03$ \\
\hline Female & 2 & $8.47 \pm 0.58$ & 2 & $8.31 \pm 0.69$ & 1 & $6.94 \pm 0.00$ \\
\hline HOMA-IR & 5 & $1.09 \pm 0.06$ & 4 & $1.86 \pm 0.45$ & 3 & $2.07 \pm 0.20$ \\
\hline Male & 3 & $1.17 \pm 0.02$ & 2 & $1.78 \pm 0.22$ & 2 & $2.27 \pm 0.01$ \\
\hline Female & 2 & $0.97 \pm 0007$ & 2 & $1.95 \pm 1.08$ & 1 & $1.67 \pm 0.00$ \\
\hline Insulin (pmol/L) & 5 & $20.05 \pm 1.39$ & 4 & $33.18 \pm 7.34$ & 3 & $42.97 \pm 2.76$ \\
\hline Male & 3 & $21.54 \pm 1.91$ & 2 & $31.14 \pm 3.50$ & 2 & $45.72 \pm 0.42$ \\
\hline Female & 2 & $17.82 \pm 0.00$ & 2 & $35.22 \pm 17.40$ & 1 & $37.47 \pm 0.00$ \\
\hline Triglycerides (mmol/L) & 5 & $0.66 \pm 0.09$ & 4 & $0.58 \pm 0.03$ & 3 & $0.83 \pm 0.16$ \\
\hline Male & 3 & $0.70 \pm 1.16$ & 2 & $0.55 \pm 0.04$ & 2 & $0.86 \pm 0.28$ \\
\hline Female & 2 & $0.60 \pm 0.08$ & 2 & $0.62 \pm 0.05$ & 1 & $0.76 \pm 0.00$ \\
\hline
\end{tabular}

Table 4. Differences in biomarkers between experimental diet groups.

\begin{tabular}{|c|c|c|}
\hline Biomarker & Group Comparison & P-value \\
\hline \multirow{3}{*}{$\mathrm{BMI}\left(\mathrm{g} / \mathrm{cm}^{2}\right)$} & Control/PUFA \& Western/PUFA & 0.905 \\
\hline & Western/PUFA \& Western/Western & 0.629 \\
\hline & Control/PUFA \& Western/Western & 0.786 \\
\hline \multirow{3}{*}{ HbA1c (\%) } & Control/PUFA \& Western/PUFA & 1.000 \\
\hline & Western/PUFA \& Western/Western & 0.229 \\
\hline & Control/PUFA \& Western/Western & 0.143 \\
\hline \multirow{3}{*}{ Fasting Glucose (mmol/L) } & Control/PUFA \& Western/PUFA & 0.730 \\
\hline & Western/PUFA \& Western/Western & 0.857 \\
\hline & Control/PUFA \& Western/Western & 0.786 \\
\hline \multirow{3}{*}{ HOMA-IR } & Control/PUFA \& Western/PUFA & 0.286 \\
\hline & Western/PUFA \& Western/Western & 0.629 \\
\hline & Control/PUFA \& Western/Western & 0.036 \\
\hline \multirow{3}{*}{ Fasting Insulin (pmol/L) } & Control/PUFA \& Western/PUFA & 0.111 \\
\hline & Western/PUFA \& Western/Western & 0.400 \\
\hline & Control/PUFA \& Western/Western & 0.036 \\
\hline \multirow{3}{*}{ Triglycerides (mmol/L) } & Control/PUFA \& Western/PUFA & 0.556 \\
\hline & Western/PUFA \& Western/Western & 0.114 \\
\hline & Control/PUFA \& Western/Western & 0.393 \\
\hline
\end{tabular}


lower among Western/PUFA animals than Western/Western offspring, these differences did not reach statistical significance. By contrast, Western/Western animals had significantly higher fasting plasma insulin $(\mathrm{P}<0.05)$, and were significantly more insulin resistant $(\mathrm{P}<0.05)$ than animals weaned to the PUFA diet, but whose mothers consumed the control diet during pregnancy-lactation (Control/PUFA) (Figure 1).

\subsection{Triglycerides}

The largest difference in mean fasting triglycerides between diet groups was the $30 \%$ higher value of Western/ Western animals compared to Western/PUFA offspring $(0.58 \mathrm{mmol} / \mathrm{L})$. Mean triglycerides levels of Control/ PUFA animals $(0.66 \mathrm{mmol} / \mathrm{L})$ were intermediate to those of Western/Western and Western/PUFA offspring. None of these differences, however, reached statistical significance.

\subsection{Body Weight}

There were no significant differences in body weights on day 7 among offspring whose mother consumed the Western or Control diets during pregnancy-lactation. By day 21 (weaning), however, offspring of mothers fed the Western diet during pregnancy-lactation were significantly $(\mathrm{P}<0.01)$ higher. Table 5 shows the average body weights at 7 and 21 days among offspring whose mothers consumed Western or Control diets during pregnancy-lactation.

Western/Western offspring maintained the highest body weights throughout the study (after weaning), weighing 31 grams more, on average, than Western/PUFA animals, and 15 grams more than Control/PUFA offspring on day 120 (Table 6). Differences in body weights from day 25 onward, however, were not statistically significant (data not shown).
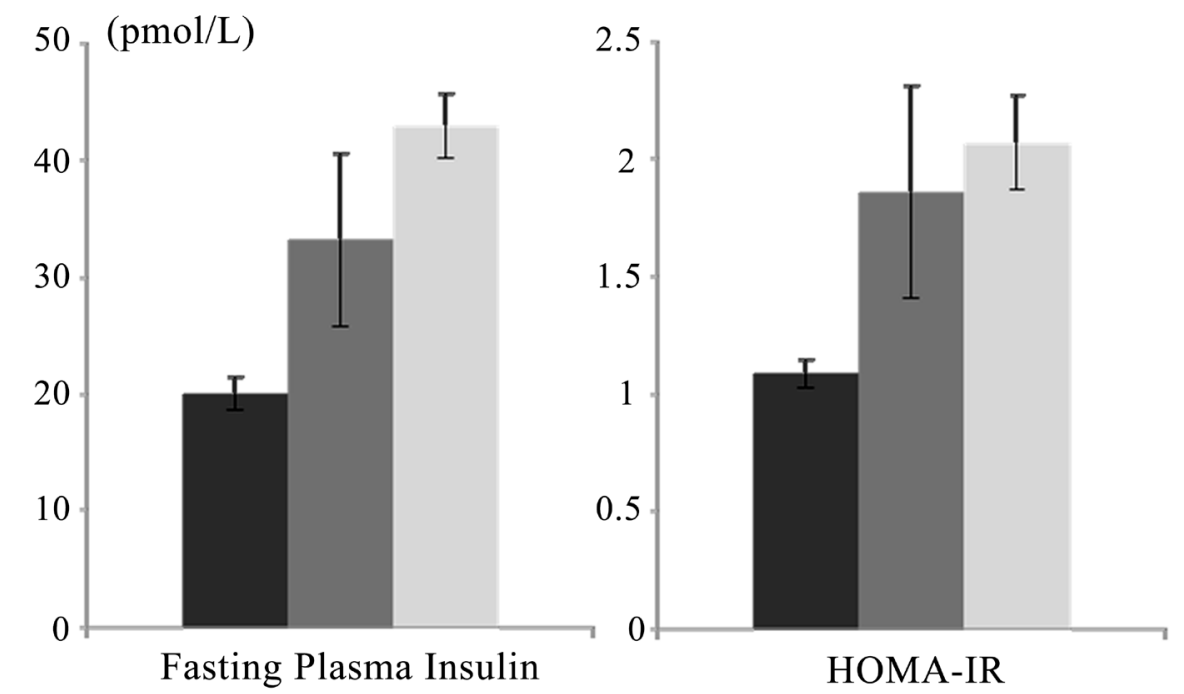

Control-PUFA Western-PUFA Western-Western

Figure 1. Fasting Plasma Insulin and Resistance as Measured by HOMA-IR at Day 120.

Table 5. Body weight in grams for pre-weaning rats by maternal diet.

\begin{tabular}{|c|c|c|c|c|c|}
\hline \multirow{2}{*}{ Day } & \multicolumn{2}{|c|}{ Control } & \multicolumn{2}{|c|}{ Western } & \multirow{2}{*}{ P-value* } \\
\hline & $\mathrm{N}$ & Mean \pm S.E. & $\mathrm{N}$ & Mean \pm S.E. & \\
\hline 7 & 18 & $17.20 \pm 0.19$ & 18 & $16.44 \pm 0.48$ & 0.153 \\
\hline 21 & 18 & $47.93 \pm 0.61$ & 18 & $51.64 \pm 0.72$ & 0.000 \\
\hline
\end{tabular}

\footnotetext{
*2 sample t-test.
} 
Table 6. Body weight in grams for post-weaning rats by experimental diet.

\begin{tabular}{ccccccc}
\hline & \multicolumn{2}{c}{ Control/PUFA } & \multicolumn{2}{c}{ Western/PUFA } & \multicolumn{2}{c}{ Western/Western } \\
\cline { 2 - 6 } Day & $\mathrm{N}$ & Mean \pm S.E. & $\mathrm{N}$ & Mean \pm S.E. & $\mathrm{N}$ & Mean \pm S.E. \\
\hline 25 & 5 & $64.54 \pm 0.91$ & 4 & $66.95 \pm 2.34$ & 3 & $69.83 \pm 4.83$ \\
49 & 5 & $180.44 \pm 6.80$ & 4 & $184.33 \pm 8.49$ & 3 & $217.47 \pm 26.23$ \\
63 & 5 & $257.34 \pm 23.10$ & 4 & $232.85 \pm 17.88$ & 3 & $277.63 \pm 42.37$ \\
70 & 5 & $278.22 \pm 22.16$ & 4 & $255.10 \pm 23.18$ & 3 & $296.83 \pm 45.07$ \\
81 & 5 & $302.62 \pm 27.59$ & 4 & $283.50 \pm 25.28$ & 3 & $321.80 \pm 45.95$ \\
109 & 5 & $341.48 \pm 32.99$ & 4 & $328.88 \pm 33.80$ & 3 & $370.57 \pm 42.75$ \\
120 & 5 & $355.50 \pm 30.60$ & 4 & $339.78 \pm 32.83$ & 3 & $370.93 \pm 48.41$ \\
\hline
\end{tabular}

\section{Discussion}

\section{Maternal Diet, Postweaning Diet, and Metabolic Health}

Previous experimental animal studies have shown that the adult offspring of mothers who consume a diet in high-saturated fat during pregnancy and lactation and are then weaned to a control diet have high levels of fasting insulin, are insulin resistant, and obese [10]. These effects are further exacerbated when these offspring are weaned to the same high-fat diet [11]. Other studies have shown that omega-3 PUFA-rich diets can improve key biomarkers of metabolic functioning in adulthood [3]. The present study extends both of these lines of research by showing, for the first time, that the metabolic benefits of a postweaning omega-3 PUFA-rich diet, represented in the current study design by the Control/PUFA diet, are tempered by deleterious developmental effects of a high-saturated fat Western maternal diet during pregnancy-lactation (modeled by the Western/PUFA group). While the Western/PUFA offspring in our study had lower mean plasma insulin, triglycerides, and HOMA-IR values than Western/Western animals, none of these differences were statistically significant, although this might be attributed to current pilot study's small size. Although the mean fasting glucose values of Control/PUFA and Western/PUFA animals were higher than Western/Western offspring, these differences were not statistically significant, and the nearly identical mean HbA1c values of the three groups suggests the lower fasting glucose levels of Western/Western animals were of short duration, and likely due to fasting insulin values that were 53\% higher than Control/PUFA animals. Only mean fasting insulin and HOMA-IR values between Control/PUFA and Western/Western off-spring reached statistical significance. These findings suggest that omega-3 PUFA-rich diets known to improve metabolic health in adulthood are likely to be unable to compensate for the developmentally programmed, metabolically deleterious effects of a high-saturated fat Western maternal diet during pregnancy and lactation.

\section{Conclusion}

\section{Study Limitations and Implications for Primary Type 2 Diabetes Prevention and Treatment}

The size of our pilot study is a significant limitation, and consequently, our results should be interpreted with caution. If confirmed by additional research, however, our results may have important implications for the content and timing of potential future diet-based interventions aimed at type 2 diabetes/Metabolic Syndrome prevention and treatment. The results presented here are consistent with a large body of experimental animal, clinical and epidemiological studies on the developmental origins of obesity related health disorders. Our findings suggest that while interventions aimed at increasing intake of omega-3 LC-PUFAs in adulthood can improve biomarkers of metabolic health, the deleterious effects of a poor (e.g., high energy, low fiber, high-saturated fat) maternal diet during pregnancy and lactation limit the effectiveness of such an intervention. As a result, future prevention efforts may be most effective by complementing adult dietary interventions with longer term, multi-generation prevention strategies that aim to optimize maternal and child health by improving maternal health and nutrition before pregnancy. 


\section{Acknowledgements}

The authors would like to acknowledge the funding support provided by the UNLV Edwards and Olswang and the Rocchio research grants. We would also like to acknowledge the helpful contributions of Alyssa Crittenden and Celeste Giordano.

\section{References}

[1] Nettleson, J.A. and Katz, R. (2005) N-3 Long-Chain Polyunsaturated Fatty Acids in Type 2 Diabetes: A Review. Journal of the American Dietetic Association, 105, 428-440. http://dx.doi.org/10.1016/j.jada.2004.11.029

[2] Jimenez-Gomez, Y., Cruz-Teno, C., Rangel-Zuñiga, O.A., Peinado, J.R., Perez-Martinez, P., Delgado-Lista, J., GarciaRios, A., Camargo, A., Vazquez-Martinez, R., Ortega-Bellido, M., Perez-Jimenez, F., Roche, H.M., Malagon, M.M. and Lopez-Miranda, J. (2014) Effect of Dietary Fat Modification on Subcutaneous White Adipose Tissue Insulin Sensitivity in Patients with Metabolic Syndrome. Molecular Nutrition \& Food Research, 58, 2177-2188. http://dx.doi.org/10.1002/mnfr.201300901

[3] Paniagua, J.A., Pérez-Martinez, P., Gjelstad, I.M., Tierney, A.C., Delgado-Lista, J., Defoort, C., Blaak, E.E., Risacrus, U., Drevon, C.A., Kiec-Wilk, B., Lovegrove, J.A., Roche, H.M. and López-Miranda, J. (2011) A Low-Fat High-Carbohydrate Diet Supplemented with Long-Chain n-3 PUFA Reduces the Risk of the Metabolic Syndrome. Atherosclerosis, 218, 443-450. http://dx.doi.org/10.1016/j.atherosclerosis.2011.07.003

[4] Liu, H.Q., Qiu, Y., Mu, Y., Zhang, X.J., Liu, L., Hou, X.H., Zhang, L., Xu, X.N., Ji, A.L., Cao, R., Yang, R.H. and Wang, F. (2013) A High Ratio of Dietary n-3/n-6 Polyunsaturated Fatty Acids Improves Obesity-Linked Inflammation and Insulin Resistance through Suppressing Activation of TLR4 in SD Rats. Nutrition Research, 33, 849-858. http://dx.doi.org/10.1016/j.nutres.2013.07.004

[5] Fickova, M., Hubert, P., Crémel, G. and Leray, C. (1998) Dietary (n-3) and (n-6) Polyunsaturated Fatty Acids Rapidly Modify Fatty Acid Composition and Insulin Effects in Rat Adipocytes. The Journal of Nutrition, 128, 512-519.

[6] Luo, J., Rizkalla, S.W., Boillot, J., Alamowitch, C., Chaib, H., Bruzzo, F., Desplanque, N., Daliz, A.M., Georges Durand, G. and Slama, G. (1996) Dietary (n-3) Polyunsaturated Fatty Acids Improve Adipocyte Insulin Action and Glucose Metabolism in Insulin-Resistant Rats: Relation to Membrane Fatty Acids. The Journal of Nutrition, 126, 1951- 1958.

[7] Peyron-Caso, E., Fluteau-Nadler, S., Kabir, M., Guerre-Millo, M., Quignard-Boulange, A., Slama, G. and Rizkalla, S.W. (2002) Regulation of Glucose Transport and Transporter 4 (GLUT-4) in Muscle and Adipocytes of Sucrose-Fed Rats: Effects of N-3 Poly- and Monounsaturated Fatty Acids. Hormone and Metabolic Research, 34, 360-366. http://dx.doi.org/10.1055/s-2002-33467

[8] Benyshek, D.C. (2007) The Developmental Origins of Obesity and Related Health Disorders: Prenatal and Perinatal Factors. Collegium Antropologicum, 31, 11-17.

[9] Popkin, B.M. (2006) Global Nutrition Dynamics: The World Is Shifting Rapidly toward a Diet Linked with Noncommunicable Diseases. The American Journal of Clinical Nutrition, 84, 289-298.

[10] Liang, C.Y., Oest, M.E. and Prater, M.R. (2009) Intrauterine Exposure to High Saturated Fat Diet Elevates Risk of Adult-Onset Chronic Diseases in C57BL/6 Mice. Birth Defects Research Part B: Developmental and Reproductive Toxicology, 86, 377-384. http://dx.doi.org/10.1002/bdrb.20206

[11] Khan, I.Y., Taylor, P.D., Dekou, V., Seed, P.T., Lakasing, L., Graham, D., Dominiczak, A.F., Hanson, M.A. and Poston, L. (2003) Gender-Linked Hypertension in Offspring of Lard-Fed Pregnant Rats. Hypertension, 41, 168-175. http://dx.doi.org/10.1161/01.HYP.0000047511.97879.FC 
Scientific Research Publishing (SCIRP) is one of the largest Open Access journal publishers. It is currently publishing more than 200 open access, online, peer-reviewed journals covering a wide range of academic disciplines. SCIRP serves the worldwide academic communities and contributes to the progress and application of science with its publication.

Other selected journals from SCIRP are listed as below. Submit your manuscript to us via either submit@scirp.org or Online Submission Portal.
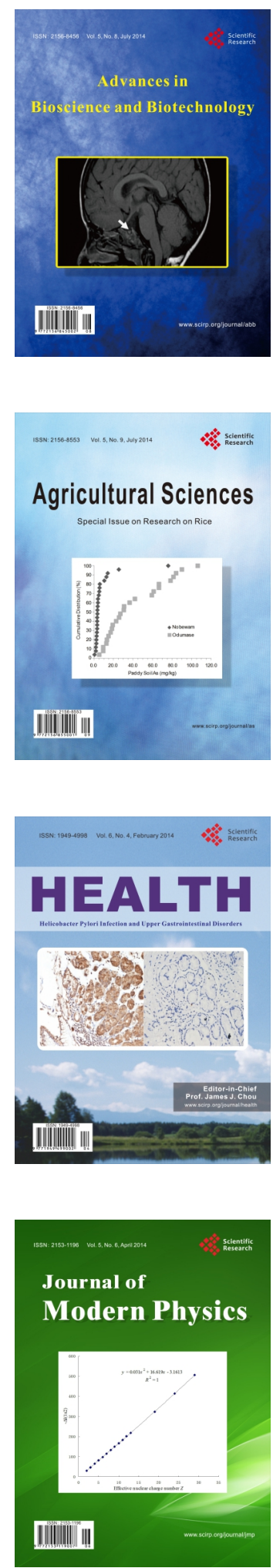
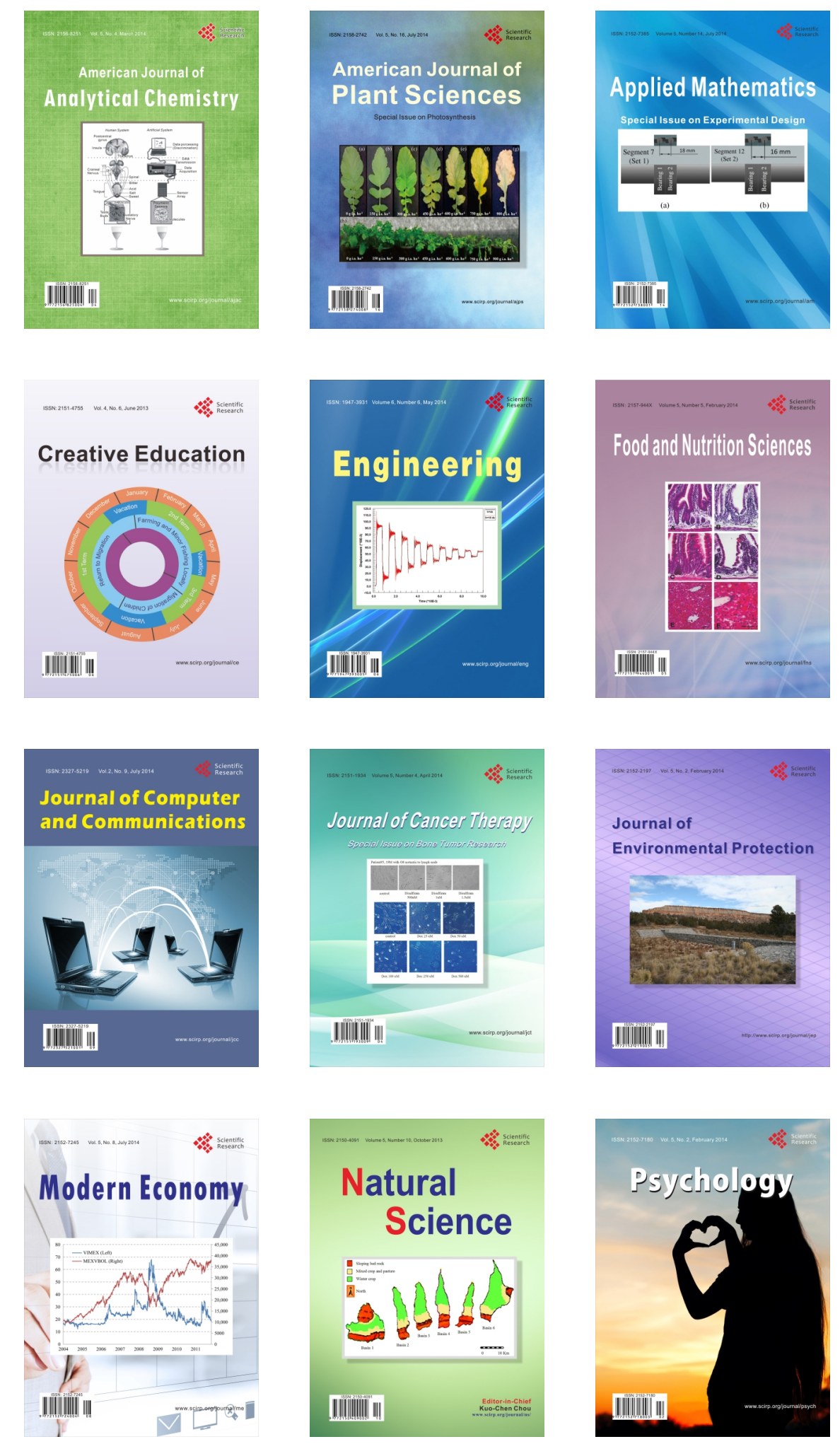